\title{
Scaled Schrodinger equation and the exact wave function
}

$\operatorname{AUTHOR}(\mathrm{S})$ :

Nakatsuji, $\mathrm{H}$

CITATION:

Nakatsuji, H. Scaled Schrodinger equation and the exact wave function. Physical Review Letters 2004, 93(3): 030403.

ISSUE DATE:

2004-07-16

URL:

http://hdl.handle.net/2433/39923

RIGHT:

Copyright 2004 American Physical Society 


\title{
Superfluid-Superfluid Phase Transitions in a Two-Component Bose-Einstein Condensate
}

\author{
Anatoly Kuklov, ${ }^{1}$ Nikolay Prokof'ev, ${ }^{2,3}$ and Boris Svistunov ${ }^{2,3}$ \\ ${ }^{1}$ Department of Engineering Science and Physics, The College of Staten Island, City University of New York, \\ Staten Island, New York 10314, USA \\ ${ }^{2}$ Department of Physics, University of Massachusetts, Amherst, Massachusetts 01003, USA \\ ${ }^{3}$ Russian Research Center "Kurchatov Institute," 123182 Moscow, Russia
}

(Received 29 May 2003; published 22 January 2004)

\begin{abstract}
Depending on the Hamiltonian parameters, two-component bosons in an optical lattice can form at least three different superfluid phases in which both components participate in the superflow: a (strongly interacting) mixture of two miscible superfluids (2SF), a paired superfluid (PSF) vacuum, and (at a commensurate total filling factor) the super-counter-fluid (SCF) state. We study the universal properties of the 2SF-PSF and 2SF-SCF quantum phase transitions and show that (i) they can be mapped onto each other and (ii) their universality class is identical to the $(d+1)$-dimensional normal-superfluid transition in a single-component liquid. The finite-temperature 2SF-PSF(SCF) transitions and the topological properties of 2SF-PSF(SCF) interfaces are also discussed.
\end{abstract}

PACS numbers: 03.75.Kk, 05.30.Jp

The recent success in experimental studies of ultracold atoms in a 3D optical lattice (OL) [1] signals a major breakthrough in the field of quantum lattice systems. The theoretical framework which describes the physics of atomic gases in an OL is given by the on-site BoseHubbard model [2]. Its seminal prediction [3]—the superfluid-Mott-insulator (SF-MI) transition-has been unambiguously confirmed [1].

Realistic experimental perspectives of trapping several atomic species in the ultraquantum regime have inspired theoretical studies of multicomponent systems in OLs [4-9]. In particular, it has been argued that the two-component commensurate mixture of inconvertible atoms can be in the super-counter-fluid (SCF) state [5]. In this state, the net atomic superfluid current is impossible, and yet the equal-current flows of two components in opposite directions are superfluid. Another intriguing superfluid ground state which exists in the twocomponent (with equal particle numbers) Bose system in OL is the paired superfluid (PSF) vacuum [7,10]. Qualitatively, this state is equivalent to the superfluid state of two-atomic molecules and a BCS superconductor. An important quantitative difference with the BCS theory is that bosonic superfluidity exists without pairing correlations too, and the PSF is always associated with finite intraspecies interaction. At the moment, it is not clear whether PSF can be realized in atomic gases without OL. At the two miscible superfluid (2SF) PSF transition point the pairing interaction is necessarily strong; i.e., the scattering length for atoms ready to form a pair is of the order of (or larger) than the interatomic separation. Under these conditions, metastable atomic gases are likely to become unstable from the experimental point of view because of very large inelastic cross sections leading to formation of fast tight molecules (not to be confused with the loose pairs we are discussing here) and fast atoms. In $\mathrm{OL}$, this recombination channel is not an issue since now the regime of strong/weak interaction is controlled by the ratio of the tunneling constant to the strength of the effective on-site interaction, while decay rates are still controlled by the one-site physics and are not sensitive to tunneling.

In this Letter, we discuss the universal properties of the 2SF-PSF and 2SF-SCF phase transitions. First, we prove that the two transitions are equivalent to each other by establishing mapping between the PSF and SCF phases. According to mapping, SCF can be viewed as a "molecular" superfluid, where "molecules" consist of particles of one-component and holes of another component. Correspondingly, the SCF transition is equivalent to the binding of atomic superfluids into PSF. Our main focus is on the quantum phase transition. We present strong arguments that this transition is in the $(d+1)$ dimensional $\mathrm{U}(1)$ universality class, and propose an effective $(d+1)$-dimensional classical model describing it. It allows us to relate correlation functions in terms of the original bosonic fields to correlators of the U(1) order parameter. In the vicinity of the quantum phase transition point, our considerations are naturally generalized to the finite-temperature case, predicting the same $\mathrm{U}(1)$ universality class (but now in $d$ dimensions) for the 2 SF-PSF(SCF) transition at $T>0$. We verify our predictions numerically by performing Monte Carlo simulations of a 3D two-component closed-loop current model of Ref. [11], with the long-range critical behavior identical to that of a two-component 2D quantum system. Finally, we note that the 2SF-PSF(SCF) phase transition preserves the molecular part of the order parameter and discuss the consequences of this fact for the structure of the vortices in hybrid systems containing $2 \mathrm{SF}-\mathrm{PSF}(\mathrm{SCF})$ interfaces.

The qualitative equivalence of PSF and SCF phases can be understood on the basis of Feynman's representation of quantum statistics in terms of particle paths (world lines) in imaginary time $\tau \in[0, \beta \rightarrow \infty)$, where $\beta$ is the inverse 
temperature. In this representation, the superfluid ground state is characterized by world lines forming macroscopic cycles (for brevity, we call them $M$ cycles), when the end of one world line at $\tau=\beta$ is the beginning of another world line at time $\tau=0$, and so on (partition function world lines in imaginary time are $\beta$ periodic). The qualitative difference between the 2SF and PSF ground states is that in PSF each $A$ component world line is bound to some $B$ component world line, and in the long-range limit there are no free single-component world lines forming independent $M$ cycles. All $M$ cycles are formed only by pairs of lines, and we arrive at the picture of PSF, or molecular superfluid. Less obvious is the fact that SCF has the same world line structure as PSF. The key observation is that for integer total filling factor one may use a hole representation for one of the components, say, component $B$. We readily understand that the only world line structure consistent with the absence of net superfluid response is when each $B$-hole world line is bound with some $A$-particle world line - this is the only possibility of forming $M$ cycles out of particle-hole pairs without having independent single-component $M$ cycles. Macroscopically, bound particle-hole pairs behave like new molecules with zero particle number charge, and their flow is equivalent to the counterflow of participating components.

In view of the SCF-PSF equivalence, in what follows we discuss PSF only, implying that all results remain valid for SCF as well.

The world line language presents also a "graphic picture" of critical fluctuations driving the PSF-2SF transition. Suppose that initially we are deep in the PSF phase. Then each $A$ line is closely followed by some $B$ line. As the coupling between components becomes weaker, bound lines demonstrate local unbinding fluctuations; see Fig. 1(a). These fluctuations can be viewed as singlecolored oriented loops, one part of the loop representing, say, an $A$ line, and the other part representing a $B$ line with the reversed direction; see Fig. 1(b). Close to the critical point, unbinding loops grow larger and start reconnecting with each other (become dense). We assume that only large-scale loops are essential for characterizing the universality class of the transition; the details of short-range behavior are simply determining parameters of the critical action for these loops. The phase transition in a system of oriented loops in $d+1$ dimensions (lead-
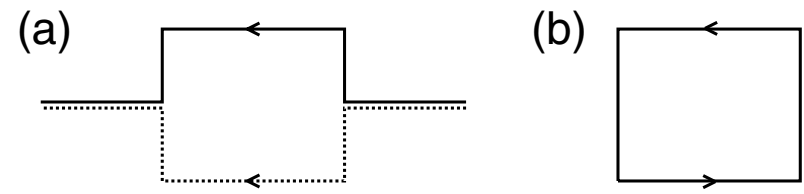

FIG. 1. (a) Unbinding fluctuations of two coupled world lines of the two-component system. (b) The single-loop effective representation. World lines of different species are depicted as solid and dotted lines, respectively. ing to the appearance of macroscopic-size loops) is known to describe the SF-MI transition in a commensurate system of bosons on a $d$-dimensional lattice (see, e.g., [11]). In its turn, this transition is equivalent to the finitetemperature phase transition between normal and superfluid states in $d+1$ dimensions [3]. Hence, the above reasoning suggests a mapping between the PSF-2SF and MI-SF transitions, and establishes that the PSF-2SF transition is in the universality class of classical $(d+1)$ dimensional U(1) models.

We now argue that in the long-wave limit our system also can be mapped onto a $(d+1)$-dimensional model of two-color classical rotators. This mapping is used to have one more argument in favor of the U(1) universality class, and to establish important relations between basic correlation functions; it also provides a natural generalization of our considerations to finite temperatures.

The presence of lattice commensurability is not crucial for the PSF-2SF transition since both phases involved are superfluid. However, it proves convenient to formally assume that we are dealing with the doublecommensurate system - filling factors of both species are integers. According to [3], commensurate $d$-dimensional lattice bosons map in the long-wave limit to a $(d+1)$ dimensional array of rotators with the Hamiltonian

$$
H=-\gamma \sum_{\langle i j\rangle} \cos \left(\Phi_{i}-\Phi_{j}\right),
$$

where $\Phi_{j} \in[0,2 \pi)$ is the angle of the $j$ th rotator [or phase of the bosonic order-parameter field $\left.\Psi(j) \sim e^{i \Phi_{j}}\right]$ and $\langle\cdots\rangle$ stands for the nearest neighbor sites on a square lattice. In our case, we need three quantum fields: $\psi_{A}$ and $\psi_{B}$ for the two components, and the field $\psi_{P}$ for the pairs. This suggests terms similar to Eq. (1) for each of the corresponding three phases. However, one also has to account for the terms in the effective Hamiltonian converting a bound pair into two atoms and vice versa. In terms of the rotator model, this leads to a local term $\propto \sum_{j} \cos \left[\Phi_{j}^{(P)}-\Phi_{j}^{(A)}-\Phi_{j}^{(B)}\right]$ reducing the symmetry of the three-color rotor system to $\mathrm{U}(1) \times \mathrm{U}(1)$. This term introduces some (loose) constraint on the difference between the phase $\Phi_{j}^{(P)}$ and the sum $\Phi_{j}^{(A)}+\Phi_{j_{B}(B)}$. Replacing it with the rigid constraint $\Phi_{j}^{(P)}=\Phi_{j}^{(A)}+\Phi_{j}^{(B)}$, we reduce the number of independent variables from three to twoas one could expect from the very beginning given the original $\mathrm{U}(1) \times \mathrm{U}(1)$ symmetry of our system. As a result, we arrive at the following Hamiltonian (for simplicity, we assume exchanging symmetry between the components):

$$
\begin{gathered}
H=-\sum_{\langle i j\rangle}\left(\gamma_{1} \cos \Phi_{i j}+\gamma_{2} \cos \Phi_{i j}^{(A)}+\gamma_{2} \cos \Phi_{i j}^{(B)}\right), \\
\Phi_{j}=\Phi_{j}^{(A)}+\Phi_{j}^{(B)}
\end{gathered}
$$

where $\Phi_{i j}=\Phi_{i}-\Phi_{j}$ and $\Phi_{i j}^{(A, B)}=\Phi_{i}^{(A, B)}-\Phi_{j}^{(A, B)}$. Apart from the 2SF-PSF transition, this model also can be used 
to describe other phase transitions in the doubly commensurate system.

It is convenient to introduce the phase difference

$$
\varphi_{j}=\left(\Phi_{j}^{(A)}-\Phi_{j}^{(B)}\right) / 2,
$$

and to rewrite the Hamiltonian (2) as $\left(\varphi_{i j}=\varphi_{i}-\varphi_{j}\right)$

$$
H=-\sum_{\langle i j\rangle}\left[\gamma_{1} \cos \Phi_{i j}+2 \gamma_{2} \cos \left(\Phi_{i j} / 2\right) \cos \varphi_{i j}\right] .
$$

(The fields $\Phi$ and $\varphi$ describe charge and pseudospin degrees of freedom, respectively.) Though the new variables $\Phi_{j}$ and $\varphi_{j}$ cannot be interpreted as the angles of new rotators - the configurational space of the original rotators $\Phi_{j}^{(A)}$ and $\Phi_{j}^{(B)}$ is exhausted with, say, $\Phi_{j} \in[0,2 \pi)$ and $\varphi_{j} \in(-\pi, \pi]$, while the Hamiltonian (5) is not $2 \pi$ periodic with respect to $\Phi_{j}$ - for our purposes it is sufficient that only $\varphi_{j}$ can be viewed as a rotator angle. Indeed, in both PSF and 2SF, the pair phase variable $\Phi_{j}$ is ordered and its local fluctuations are not relevant to the criticality of the 2SF-PSF transition. Therefore, we may simply set $\Phi_{j} \equiv 0$ in Eq. (5) which brings us to the effective one-component rotor model for $\varphi$ :

$$
H_{2 \mathrm{SF}-\mathrm{PSF}}=-2 \gamma_{2} \sum_{\langle i j\rangle} \cos \varphi_{i j}
$$

The transition thus is the superfluid-normal-fluid transition in the $\varphi$-channel (which means localization in the pseudospin sector); the corresponding complex order parameter is $\psi(\mathbf{X}) \propto e^{i \varphi(\mathbf{X})}$, where $\mathbf{X}$ is the space-time radius vector treated as a continuous variable in the longwave limit. Given this order parameter and Eq. (4) relating $\varphi$ to the original phases $\left(\Phi^{(A)}=-\Phi^{(B)}=\varphi\right)$, we immediately find the critical behavior of various correlation functions:

$$
\begin{aligned}
\left\langle\psi_{\mathrm{A}}^{\dagger}(X) \psi_{\mathrm{A}}(0)\right\rangle & \sim\left\langle\psi_{\mathrm{B}}^{\dagger}(0) \psi_{\mathrm{B}}(X)\right\rangle \sim\left\langle\psi_{\mathrm{A}}(0) \psi_{\mathrm{B}}(X)\right\rangle \\
& \sim\left\langle\psi^{\dagger}(X) \psi(0)\right\rangle .
\end{aligned}
$$

The model (6) with large but finite size in the $\tau$ direction describes the initial part of the finite-temperature 2SF-PSF line in the vicinity of the quantum critical point. We thus establish the universality class - $\mathrm{U}(1)$ in $d$ dimensions - for the finite-temperature II-order 2SF-PSF transition. Since the order parameter for the transition is $\sim e^{i \varphi}$ (molecular channel is not critical), we arrive at a rather counterintuitive conclusion that with increasing temperature the transition is from 2SF to PSF. The relations (7) take place at the finite-temperature critical point as well. Note, that a finite-temperature SCF phase has been argued to exist also in a planar $\mathrm{U}(1) \times \mathrm{U}(1)$ superconductor [12], caused, however, by an absolutely different microscopic mechanism.

The finite-temperature 2SF-PSF transition survives even when the two components have slightly different densities and the ground state is inevitably 2SF (both $\left\langle\psi_{A}\right\rangle$ and $\left\langle\psi_{B}\right\rangle$ are nonzero). Away from the quantum critical point, the 2 SF-PSF transition can be viewed as the superfluid to normal fluid transition in the (dilute) subsystem of excessive particles.

Equation (5) is also useful for understanding the structure of vortices across the interface between the PSF and 2SF phases. Experimentally, interfaces naturally arise in inhomogeneous systems (in a confining potential, particle densities drop to zero at the boundary, and, e.g., the SCF phase, which requires commensurability, may not survive at the edge). Suppose one creates a vortex in a PSF phase and then adiabatically removes OL and the trapping potential to observe the system by the standard technique of absorption imaging [13]. When the lattice potential is turned off, the system behaves as two weakly interacting gases. The question now is the following: Do vortices in the PSF phase transform (and how) into vortices in the resulting weakly interacting system [14]?

System inhomogeneity implies that at intermediate stages of the potential turning off, there is an interface similar to the one shown in Fig. 2(a). Ultimately, the interface shrinks and disappears with the PSF phase, while the topological structure of the 2SF state remains the same as it was when the interface existed. To understand this structure we resort to the rotator model (5). In both PSF and 2SF the phase field $\Phi$ is ordered and thus the circulation of $\nabla \Phi$ does not change across the interface. Since the phase field $\varphi$ is disordered inside the PSF phase, there are no topological constraints associated with it. Taking into account (3), we arrive at the following rule for topological charges:

$$
I^{(A)}+I^{(B)}=I,
$$

where $I^{(A, B)}$ and $I$ are integer topological charges in $2 \mathrm{SF}$ and PSF correspondingly,

$$
I^{(A, B)}=\frac{1}{2 \pi} \oint_{C_{2}} \nabla \Phi^{(A, B)} d \mathbf{l}, \quad I=\frac{1}{2 \pi} \oint_{C_{1}} \nabla \Phi d \mathbf{l} .
$$

We see that vortices in PSF always induce vortices in the 2SF phase fields $\Phi^{(A)}$ and/or $\Phi^{(B)}$, and thus make it possible to observe the circumstantial evidence of the PSF vortex in the absorption image of the weakly interacting 2SF state. However, the values of topological charges are not unambiguously defined. For example, if
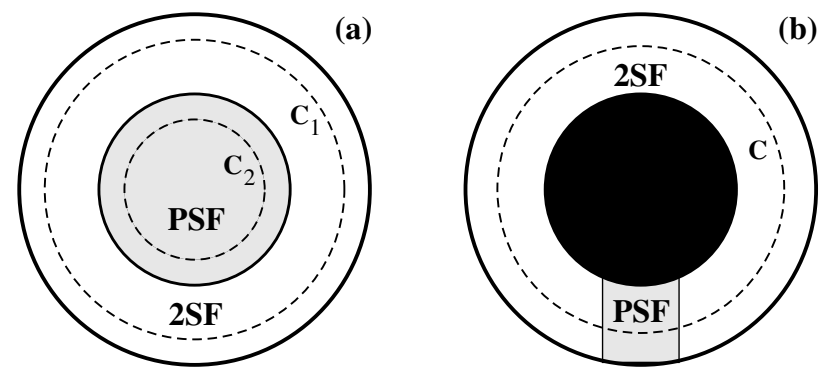

FIG. 2. Different geometries of PSF-2SF interfaces. (See discussion in the text.) 
$I=1$ then $\left(I^{(A)}=1, I^{(B)}=0\right)$ and $\left[I^{(A)}=0, I^{(B)}=1\right]$ are consistent with Eq. (8), as well as, say, $\left[I^{(A)}=2, I^{(B)}=\right.$ $-1]$. This implies that particular values of $I^{(A)}$ and $I^{(B)}$ depend on details of the experimental setup determining the lowest energy configuration. For example, if the two components have different superfluid stiffnesses and, initially, there was one vortex in the PSF, then, after creating the interface and removing the PSF, the vortex will reside in the component with lower stiffness.

Another interesting geometry is shown in Fig. 2(b). Using arguments identical to those presented above, we see that the only integer topological charge on contour $C$ is $I$. While the sum of the integrals for $I^{(A)}$ and $I^{(B)}$ still equals $I$, separately they are ill defined on $C$, because the phase $\varphi$ experiences large zero-point fluctuations in the PSF region. Suppose, then, that initially there was no PSF phase at all, and the topological charges of components were, say, $I^{(A)}=1$ and $I^{(B)}=0$. Imposing OL to create PSF eliminates quantization for the individual phases $\Phi^{(A, B)}$, while preserving the sum $I^{(A)}+I^{(B)}=1$. Accordingly - since no memory about the initial values $I^{(B)}, I^{(A)}$ is retained-further removal of the OL may result in the final $2 \mathrm{SF}$ state with $I^{(A)}=0, I^{(B)}=1$. Similar to the previous setup, if the two components have different superfluid stiffnesses, then, after the cycle of switching on and off OL, the circulation will reside in the component with lower stiffness.

There are several options to verify the above considerations numerically. One is a direct simulation of the two-component $d$-dimensional Bose-Hubbard Hamiltonian at very low temperature. However, the universality class of the phase transition and the relevant long-wave description of critical fluctuations may also be obtained for the $(d+1)$-dimensional classical lattice model built on particle trajectories in discrete imaginary time. One of the quantum-to-classical mappings for the Bose-Hubbard Hamiltonian-the $J$-current model-was developed in Ref. [11], and we straightforwardly generalized it to the two-component case. Our choice to simulate the classical action was motivated only by reasons of numeric efficiency. Recently developed quantum and classical worm algorithms do not suffer from critical slowing down [15], but the classical one is superior because of its simplicity (it was already successfully applied to the disordered onecomponent $J$-current model [16]). We defer details of simulations performed for the $d+1=3$ case to a longer paper [17] and simply mention here the results. The correlation radius and the correlation function exponents for the 2SF-PSF transition agreed with the known values for the 3D U(1)-universality class [18] within 1\%-2\% accuracy. We have directly verified that nontrivial relations between the correlation functions given by Eq. (7) hold true at the critical point, and deviations from Eq. (7) are barely visible even at distances as small as five lattice constants. We have observed the qualitative prediction of the model (6) about the transition from 2SF to PSF with increasing temperature.

To summarize, we have shown that two strongly correlated phases of the two-component bosonic system-the superfluid state of pairs and the counterflow superfluidare macroscopically equivalent. We have presented arguments supported by the results of numeric simulations that quantum phase transitions leading to formation of these phases from the state of two miscible superfluids are in the universality class of superfluid-Mott-insulator transition in a single-component bosonic system. The finite-temperature 2SF-PSF(SCF) transitions are in the universality class of a single-component superfluidnormal-fluid transition. The proposed two-color rotator model correctly describes the critical behavior of various correlators and - in the spatially inhomogeneous caseyields a simple rule for "sewing" topological defects across the boundary between the phases.

B.S. acknowledges support from The Netherlands Organization for Scientific Research (NWO). A. K. acknowledges support by PSC CUNY research program.

[1] M. Greiner et al., Nature (London) 415, 39 (2002).

[2] D. Jaksch et al., Phys. Rev. Lett. 81, 3108 (1998).

[3] M. P. A. Fisher, P. B. Weichman, G. Grinstein, and D. S. Fisher, Phys. Rev. B 40, 546 (1989).

[4] E. Demler and F. Zhou, Phys. Rev. Lett. 88, 163001 (2002).

[5] A. B. Kuklov and B.V. Svistunov, Phys. Rev. Lett. 90, 100401 (2003).

[6] G.-H. Chen and Y.-S. Wu, Phys. Rev. A 67, 013606 (2003).

[7] B. Paredes and J. I. Cirac, Phys. Rev. Lett. 90, 150402 (2003).

[8] L.-M. Duan, E. Demler, and M. D. Lukin, Phys. Rev. Lett. 91, 090402 (2003).

[9] A. E. Meyerovich, Phys. Rev. A 68, 051602 (2003).

[10] M. Yu. Kagan and D. V. Efremov, Phys. Rev. B 65, 195103 (2002).

[11] M. Wallin, E. S. Sorensen, S. M. Girvin, and A. P. Young, Phys. Rev. B 49, 12115 (1994).

[12] E. Babaev, cond-mat/0201547.

[13] M. R. Andrews et al., Science 275, 637 (1997).

[14] The nontriviality of this question for the molecular superfluid made of a single atomic bosonic species was pointed out by M. Holland (private communication).

[15] N.V. Prokof'ev, B.V. Svistunov, and I. S. Tupitsyn, Sov. Phys. JETP 87, 310 (1998); N.V. Prokof'ev and B.V. Svistunov, Phys. Rev. Lett. 87, 160601 (2001).

[16] F. Alet and E.S. Sørensen, Phys. Rev. E 67, 015701 (2003); N.V. Prokof'ev and B.V. Svistunov, Phys. Rev. Lett. 92, 015703 (2004).

[17] A. Kuklov, N. Prokof'ev, and B. Svistunov (to be published).

[18] J. A. Lipa et al., Phys. Rev. Lett. 76, 944 (1996). 